\title{
Isolation and safety characterisation of lactobacilli strains with antimicrobial properties as potential probiotics for human use
}

\begin{abstract}
The present study focuses on the antimicrobial activity and safety aspect of lactobacilli strains isolated from the anogenital region. Sixty-two lactobacilli strains were isolated from 52 healthy, non-menstruating women from a local health clinic in Puchong, Selangor, Malaysia and confirmed by $16 \mathrm{~S}$ rDNA sequencing. The samples were collected by sterile cotton swabs and directly streaked onto De Man, Rogosa, Sharpe agar prior to transportation to the lab on ice. A selective preliminary screening against several Candida albicans and Candida glabrata strains identified 5 promising lactobacilli strains for the subsequent assays; L. delbreuckii 45E, L. fermentum 28E, L. mucosae 28C, L. reuteri 29A and L. reuteri 29B. A follow-up antibacterial assay identified L. delbreuckii 45E, L. reuteri 29A and L. reuteri 29B as the lactobacilli strains with the highest inhibitory activity. Subsequent assays including haemolytic activity, susceptibility to antibiotics, quantification of D/l-lactic acid and $\mathrm{H} 2 \mathrm{O} 2$ production as well as bile salt hydrolase (BSH activity) indicated that L. reuteri 29B was a promising probiotic candidate. An acute toxicity study in vivo involving the administration of $1 \times 108,1$ $\times 109$ and $1 \times 1010 \mathrm{cfu} / \mathrm{mouse} /$ day demonstrated that L. reuteri 29B did not exert any adverse effects towards the mice which were evident through the absence of pathological changes in the histological examination and blood test. There was also a lack of bacterial translocation as confirmed by Random Amplified Polymorphic DNA (RAPD). The present study highlights the possibility of utilising L. reuteri $29 \mathrm{~B}$ as a probiotic. It also demonstrates the possibility of isolating potential probiotics from the anogenital region.
\end{abstract}

Keyword: Lactobacilli; Lactobacillus reuteri; Anogenital region; Probiotic 